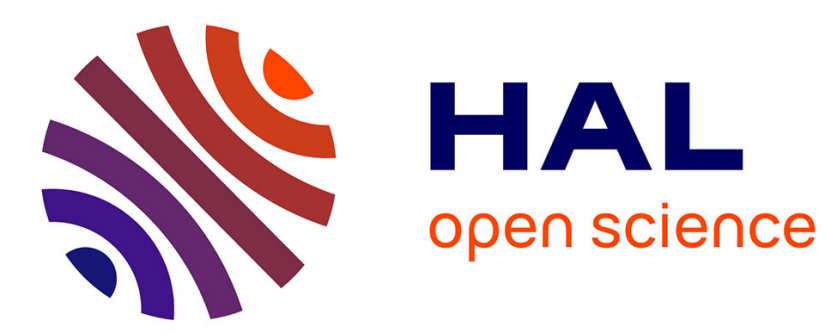

\title{
Perfectionnements apportés aux magnétomètres à résonance magnétique nucléaire à pompage électronique
}

\author{
A. Salvi
}

\section{To cite this version:}

A. Salvi. Perfectionnements apportés aux magnétomètres à résonance magnétique nucléaire à pompage électronique. Revue de Physique Appliquée, 1970, 5 (1), pp.131-134. 10.1051/rphysap:0197000501013100 . jpa-00243345

\section{HAL Id: jpa-00243345 https://hal.science/jpa-00243345}

Submitted on 1 Jan 1970

HAL is a multi-disciplinary open access archive for the deposit and dissemination of scientific research documents, whether they are published or not. The documents may come from teaching and research institutions in France or abroad, or from public or private research centers.
L'archive ouverte pluridisciplinaire HAL, est destinée au dépôt et à la diffusion de documents scientifiques de niveau recherche, publiés ou non, émanant des établissements d'enseignement et de recherche français ou étrangers, des laboratoires publics ou privés. 


\title{
PERFEGTIONNEMENTS APPORTÉS AUX MAGNÉTOMÈTRES A RÉSONANGE MAGNÉTIQUE NUGLÉAIRE A POMPAGE ÉLEGTRONIQUE
}

\author{
Par A. SALVI, \\ Centres d'Études Nucléaires, 38-Grenoble (France), Division L.E.T.I. « Magnétométrie ».
}

1. Introduction. - Les progrès réalisés dans la technique de mesure des champs magnétiques faibles, par l'intermédiaire de la résonance magnétique nucléaire à pompage électronique, sont dus en grande partie aux efforts d'adaptation de cet appareillage à bord d'un avion porteur. En effet, le déplacement rapide d'un aéronef, dans le champ magnétique terrestre, conduit à une plage de fonctionnement importante. De plus, la position quelconque de la sonde dans le champ à mesurer suppose une insensibilité du résultat quant à la direction de ce champ, par rapport à la sonde. L'environnement électromagnétique (bruits aléatoires, impulsions de toutes sortes, champs électromagnétiques intenses à diverses fréquences) nécessite de masquer la surface importante de la bobine de mesure, accordée à la fréquence de précession $(\simeq 2 \mathrm{kHz}$ pour $0,5 \mathrm{Oe})$.

2. Principe général de la mesure. - La résonance nucléaire dans un liquide peut être représentée très schématiquement par l'image gyroscopique de Bloch et définie par un système d'équations différentielles, établies en mécanique classique. Dans cette représentation et après perturbation, l'aimantation $\mathbf{M}$ des noyaux exécute un mouvement de précession autour du champ à mesurer $\mathbf{H}$. Des constantes $T_{1}$, temps de relaxation longitudinal, et $T_{2}$, temps de relaxation transversal, à peu près identiques et supérieures à la seconde, expliquent la finesse de la raie de résonance et confèrent au système une grande précision. La mesure du champ magnétique revient à une mesure de la fréquence de précession d'après la relation de Larmor :

$$
F=\frac{\gamma}{2 \pi}|\mathbf{H}| \text {. }
$$

Le rapport gyromagnétique $\gamma \mathrm{du}$ proton est une constante physique indépendante de tout effet extérieur tel que la température ou la pression. Elle a pour valeur:

$$
\gamma=2,675123 \times 10^{4} \mathrm{rad} / \mathrm{G} . \mathrm{s} \quad\left(\text { à } 8 \times 10^{-6} \text { près }\right) .
$$

La polarisation naturelle d'un ensemble $N$ de protons soumis à un champ de 0,5 gauss est excessivement faible. Rappelons que la polarisation est définie par le facteur de polarisation :

$$
P=\frac{n^{+}-n^{-}}{N} \simeq 3 \times 10^{-10}
$$

$n^{+}$étant le nombre de protons parallèles au champ $H$ et $n^{-}$le nombre de protons anti-parallèles au champ. Le signal correspondant à la résonance est perdu dans le bruit. Il est donc nécessaire d'augmenter la polarisation, afin d'obtenir un signal exploitable.

3. Polarisation dynamique. - Le principe de la polarisation dynamique, prévu par A. Abragam dès 1955, puis expérimenté par A. Abragam, I. Solo- mon, J. Combrisson en 1957 [1], met en œuvre le couplage dipolaire entre le spin $I$ du noyau d'un solvant (proton dans notre cas) et le spin $S$ d'un électron appartenant à un radical libre que l'on a dissous dans ce solvant.

Le coefficient de polarisation $P$ que l'on peut chiffrer par le rapport $\frac{\left\langle I_{z}\right\rangle}{I_{0}}$ dans lequel $I_{0}$ est la polarisation moyenne des noyaux à l'équilibre thermique et $\left\langle I_{z}\right\rangle$ la polarisation obtenue par pompage en saturant à l'aide d'un champ radiofréquence une transition permise de l'ion considéré, ce rapport peut, dans le cas d'un radical libre de type nitroxyde présentant une structure hyperfine, atteindre théoriquement des valeurs comprises entre 4000 et 5000 , tout en inversant, suivant la transition saturée, la résultante macroscopique des noyaux.

L'équation régissant un tel couplage s'écrit [1], [2] :

$$
\left\langle I_{z}\right\rangle=I_{0}-f_{\rho}\left(\left\langle S_{z}\right\rangle-S_{0}\right)
$$

relation dans laquelle $f$ représente le coefficient de fuite et s'exprime par :

$$
f=1-\frac{T_{1 \mathrm{~s}}}{T_{10}}
$$

- $T_{1 \mathrm{~s}}$ étant le temps de relaxation du solvant en présence du radical libre,

- $T_{10}$ le temps de relaxation du solvant pur,

- $\rho$ est un coefficient sans dimensions dépendant du type de couplage entre $I$ et $S$, dans notre cas, où le couplage est de type dipôle-dipôle magnétique $\rho=1 / 2$,

- $S_{0}$ représente la polarisation moyenne de l'électron à l'équilibre thermique; $\left\langle S_{z}\right\rangle$ en présence du champ radiofréquence, saturant une transition permise.

Le premier radical libre employé pour ce genre d'expérience fut le sel de Frémy ou nitro-disulfonate de peroxylamine $\mathrm{N}_{0}$ (SO3)2R2. Bien que ce radical permette des coefficients de polarisation remarquables, deux inconvénients majeurs en empêchent l'emploi dans un magnétomètre opérationnel :

1) Sa stabilité fonction de la température;

2) Sa solubilité (solvant unique $H_{0}^{2}$ ).

Le concours du laboratoire de chimie organique physique du C.E.N.-G., dirigé par M. Rassat, nous a permis d'obtenir deux radicaux libres nouveaux : le tano et le D.T.B.N. Plus stable et plus facile de préparation, c'est le premier que nous avons utilisé. Le tano au triacétone-amine-nitroxyde présente l'avantage d'une stabilité remarquable. Des échantillons préparés par R. Besson en 1962 et contrôlés périodiquement ont conservé toutes leurs propriétés; de plus, il est soluble dans une grande quantité de solvants. 
Le tano, comme le sel de Frémy, possède une structure hyperfine due au couplage d'un spin électronique $1 / 2$ au spin nucléaire $\mathbf{K}$ de l'azote; il existe donc $2(2 K+1)$ niveaux d'énergie de l'ion paramagnétique représentés par la figure 1 (voir fig. 1 in fine).

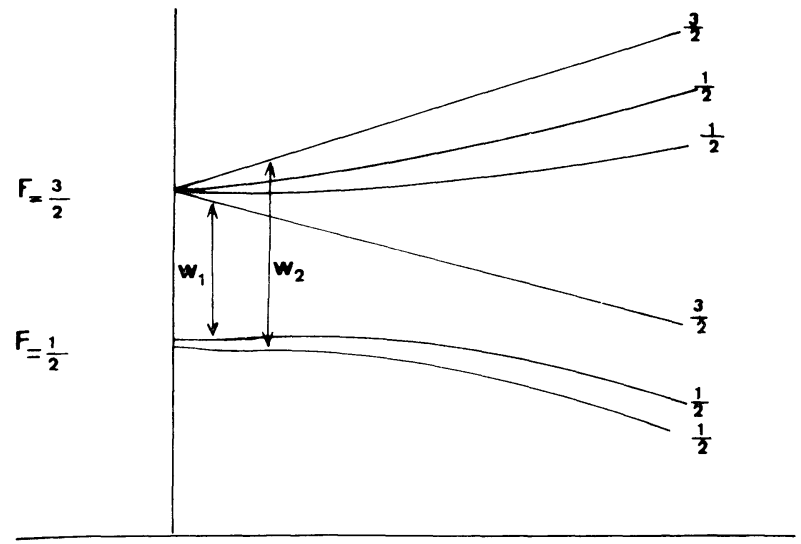

FIG. 1.

La règle de sélection $\Delta F m=\mp 1$ nous donne en champ très faible $(0,5 \mathrm{Oe})$ deux transitions permises $W_{1}$ et $W_{2}$, donnant lieu à une polarisation dynamique importante. Un calcul approché à partir des travaux de Landesman [2] donne pour le rapport théorique :

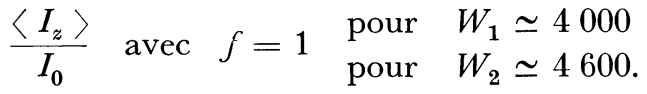

Ainsi, suivant qu'on sature par un champ radiofréquence approprié les transitions $W_{1}$ ou $W_{2}$, on observe entre les signaux de résonance nucléaire obtenus dans chacun des cas un déphasage de $180^{\circ}$ correspondant à l'inversion de la résultante macroscopique des protons du solvant (voir fig. 2 in fine). C'est cette inversion que nous mettrons en œuvre dans la réalisation de la sonde uniaxiale.

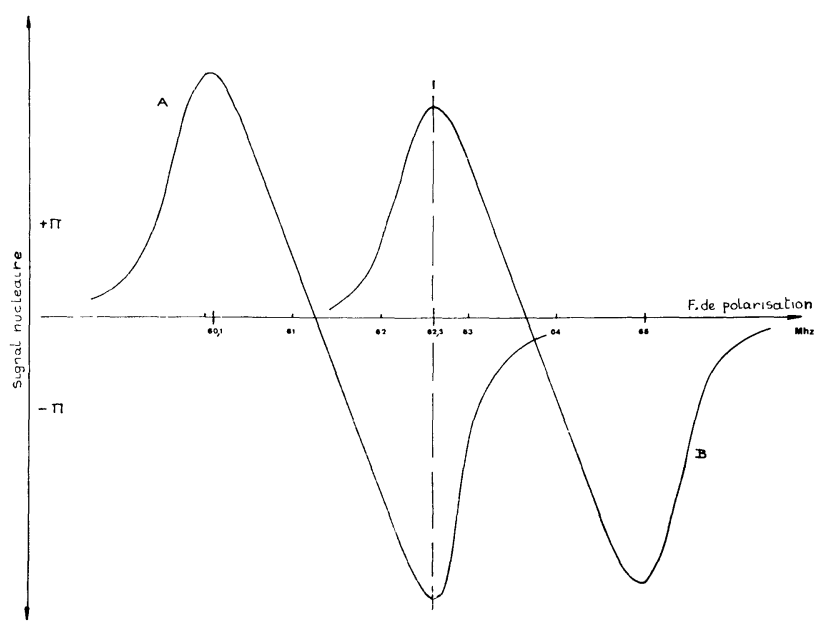

FIG. 2. - Structure électronique : radical, tano; A, D.M.E. et $B$, méthanol.

4. Effets des solvants. - Comme nous l'avons dit, le tano est un radical libre idéal, il est soluble dans un grand nombre de liquides organiques. Plus de 200 solvants ont été testés par R. Besson et M. Vincendon afin de choisir ceux qui, par leurs caractéristiques physiques d'abord (point de congélation, d'ébullition, viscosité) et par la qualité du facteur de polarisation dynamique obtenu dans chacun d'eux, pouvaient entrer dans la conception d'un magnétomètre opérationnel. Ces tests ont démontré l'action du solvant sur les fréquences de résonance électronique. Cet entraînement de fréquence est lié directement à la polarité du solvant [3]. On remarque également, en champs faibles, une modification de l'écart hyperfin. Ainsi, à $0,5 \mathrm{Oe}$, les raies

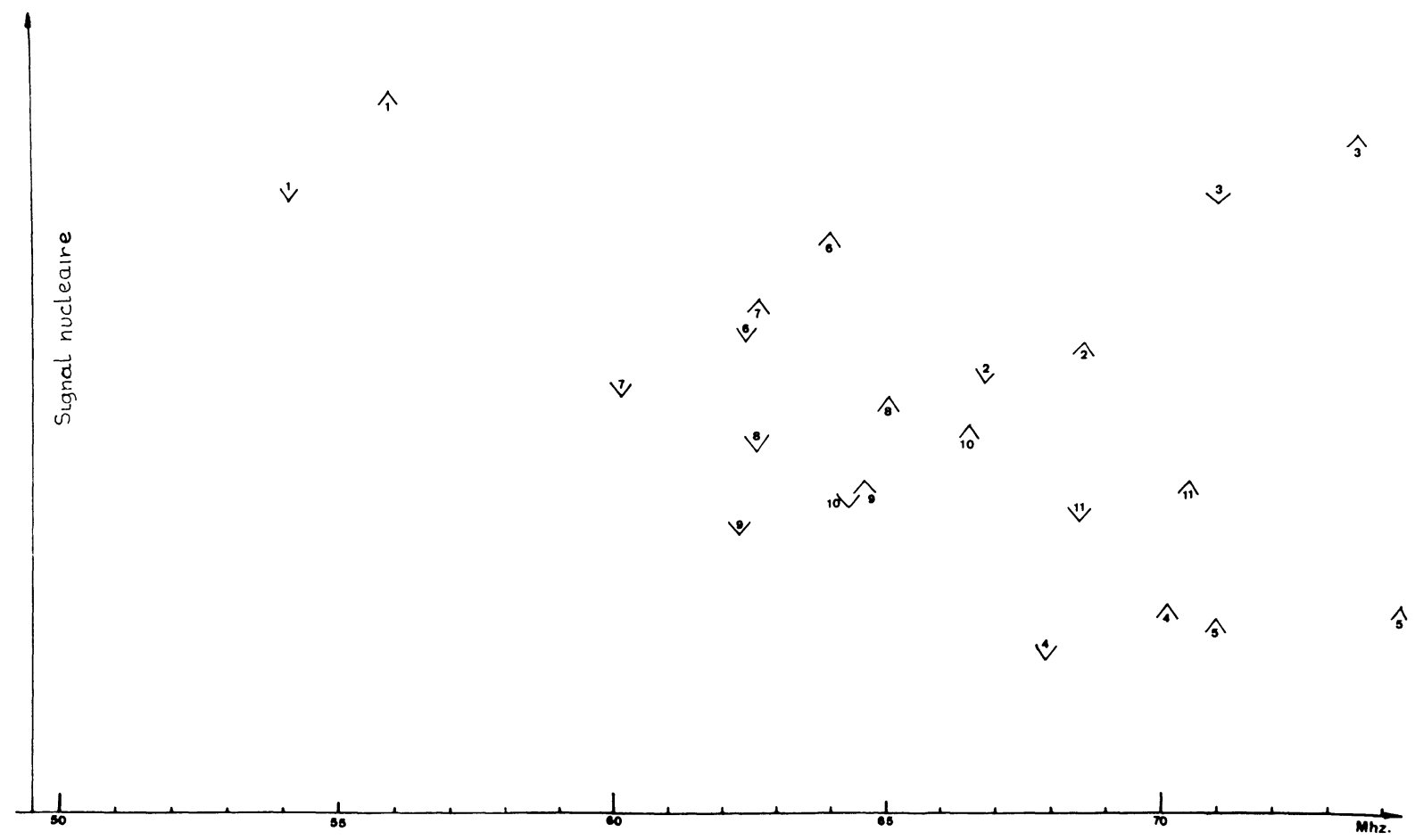

Fig. 3. - Fréquences de polarisation. Effet de solvants. 
de résonance électronique du tano à concentration de $\frac{M}{1000}$ se situent en fréquence :

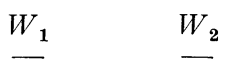

$\mathrm{H}_{2} \mathrm{O}$ désoxygé-

née

$66,8 \mathrm{MHz} \quad 68,6 \mathrm{MHz} \rightarrow$ écart $1,8 \mathrm{MHz}$

D.M.E. désoxygénée

$60,1 \mathrm{MHz} \quad 62,6 \mathrm{MHz} \rightarrow$ écart $2,5 \mathrm{MHz}$

Get entraînement de fréquence nous a été d'un grand secours quant à la réalisation de notre nouvelle sonde. Les deux solvants retenus sont le dimethoxyéthane (D.M.E.) et le méthanol, dont les caractéristiques sont les suivantes (voir fig. 3) :

D.M.E. MÉthaNol

$$
\begin{array}{lllll} 
& & - & & - \\
W_{1} & \ldots \ldots & 60,1 \\
W_{2} & \ldots \ldots & 62,6 \\
& 62,6 & \swarrow & 65,1
\end{array}
$$

4.1. Coefficient DE FUITE EXPÉRIMENTAL : GORREsPONDANT AU SIGNAL DE RÉSONANGE NUGLÉAIRE OPTIMUM. - Nous avons vu que le rapport $\frac{\left\langle I_{z}\right\rangle}{I_{0}}$ peut atteindre théoriquement 4600 si le coefficient de fuite $f=1$. Nous avons entrepris de mesurer $f$ expérimentalement en mesurant les temps de relaxation du solvant pur, puis en présence de radical à la concentration donnant le signal nucléaire maximum $\propto$ champ excitateur $H_{1}$ constant. Nous avons relevé (R. Besson) :

$$
\text { D.M.E. } \text { pur }=T_{10}=7 \mathrm{~s}
$$

$$
\begin{aligned}
\text { D.M.E. }+\operatorname{tano} \frac{M}{1600} T_{1 \mathrm{~s}} & =3,4 \mathrm{~s} . \\
\mathrm{H}_{2} \mathrm{O} \text { pure } T_{10} & =3,1 \mathrm{~s} \\
\mathrm{H}_{2} \mathrm{O}+\operatorname{tano} \frac{M}{1600} T_{1 \mathrm{~s}} & =1,5 \mathrm{~s} .
\end{aligned}
$$

Dans les deux cas nous trouvons $f \simeq 0,5$.

Gela paraît explicable si l'on considère que l'amplitude maximum du signal nucléaire est à la fois fonction du coefficient de polarisation dynamique et de la largeur de raie nucléaire en présence de radical.

5. Sonde uniaxiale à double effet. - L'idée de base était de réaliser un montage permettant de mettre en évidence la précession nucléaire des protons du solvant avec un bon rapport signal-bruit, tout en ayant une bobine de mesure à coefficient de qualité faible, afin d'obtenir une large plage d'auto-oscillation. Il fallait aussi que la sonde soit insensible à toutes les perturbations électriques ou électromagnétiques ambiantes, pouvant engendrer directement dans le bobinage de mesure des forces électromotrices parasites. Cies avantages ont été obtenus par le dispositif que nous allons vous présenter et qui exploite les d€ux transitions du

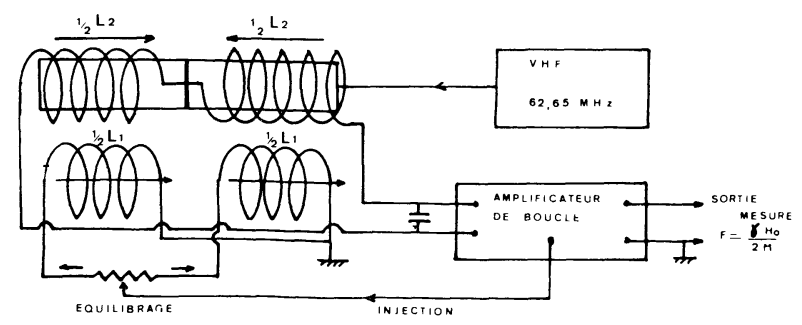

FIG. 4. - Sonde et électronique associée. tano. Cela grâce à l'entraînement de fréquence procuré par les solvants (fig. 4).

L'échantillon central est constitué de deux demi-flacons I et II, contenant pour I du méthanol et II du D.M.E., dans lesquels du tano a été dissous en proportion $M / 1000$. L'ensemble est soumis au champ magnétique V.H.F. d'une cavité coaxiale accordée à la fréquence $62,6 \mathrm{MHz}$, qui sature la transition $W_{1}$ pour I et $W_{2}$ pour II. L'inversion de $\pi$ des signaux de résonance nucléaire obtenue dans chacun des deux demi-flacons permet le montage en opposition des deux demi-bobines de mesure $1 / 2 L_{2}$ (qui doivent avoir des caractéristiques absolument identiques). L'injection du champ $H_{1}$ s'effectue par l'intermédiaire des deux demi-bobines $1 / 2 L_{1}$ dans lesquelles les courants sont équilibrés par un potentiomètre. Nous pouvons ainsi, en dehors de la résonance, rendre nul le couplage entre $L_{1}$ et $L_{2}$, celui-ci n'apparaissant qu'au moment où le phénomène de résonance nucléaire s'établit. Pour cela, il suffit de boucler $L_{2}$ à $L_{1}$ par l'intermédiaire d'un amplificateur à gain défini pour obtenir une oscillation entretenue à la fréquence de Larmor. L'en-

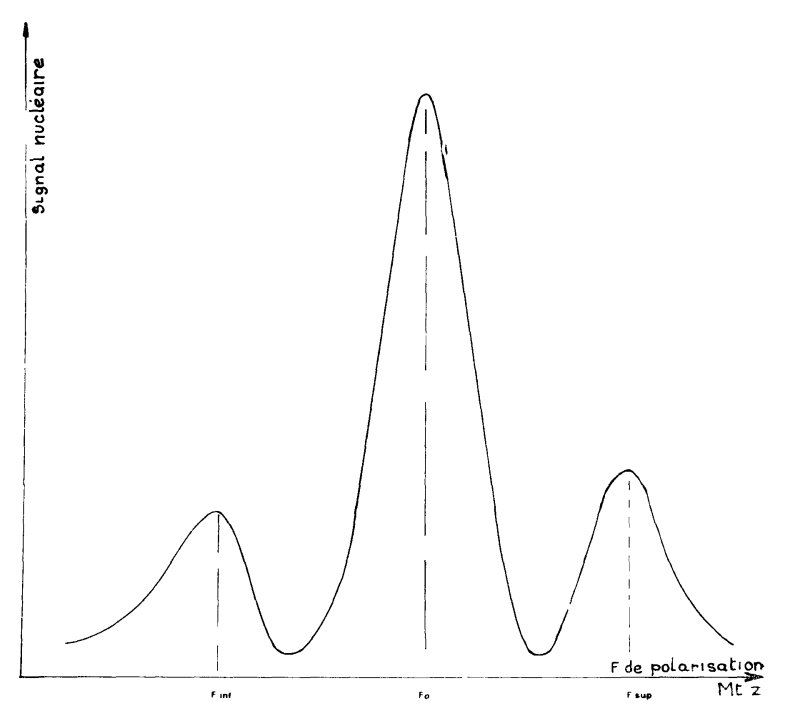

FIG. 5. - Sonde coaxiale : tano $M / 1000$ : D.M.E. et méthanol.

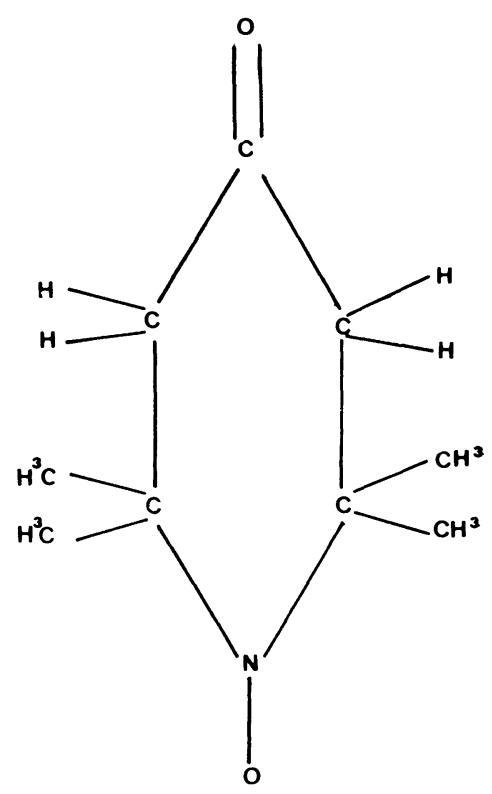

FIG. 6. - Tano classique. 
semble fonctionne en oscillateur nucléaire à couplage par spin. Pour tous les champs électromagnétiques extérieurs, les f.e.m. engendrées dans les deux demibobines $1 / 2 L_{2}$ sont en opposition et s'annulent systématiquement.

La figure 5 montre les signaux nucléaires délivrés par la sonde en fonction de la fréquence d'excitation V.H.F.

6. Tano deutéré. - Bien que parfaitement désoxygénées, les solutions de tano classique dans $\mathrm{H}_{2} \mathrm{O}$ présentent des largeurs de raies électroniques supérieures à celles du sel de Frémy, dans les mêmes conditions. En collaboration avec le laboratoire G.O.P. du G.E.N.-G., nous avons essayé d'éclaircir ce phénomène et si possible d'y remédier.
La molécule tano (voir fig. 6) comporte 16 hydrogènes dont les noyaux se couplent (magnétiquement) à l'électron libre, et, de ce fait, peuvent élargir son spectre. Après plusieurs mois de patients travaux, Chiarelli, sous la direction de A. Rassat, a pu obtenir un tano deutéré dans lequel tous les protons sont remplacés par des deutons. Le spectre de ce nouveau radical se rapproche comme prévu de celui du sel de Frémy et donne par rapport au tano normal un coefficient de polarisation dynamique supérieur de $30 \%$, avec une diminution de la puissance d'excitation V.H.F. à saturation, de l'ordre de $40 \%$ dans certains solvants (voir fig. 7).

Ge procédé sera appliqué à d'autres radicaux libres (D.T.B.N. en particulier) afin d'améliorer si possible le coefficient de polarisation dynamique.

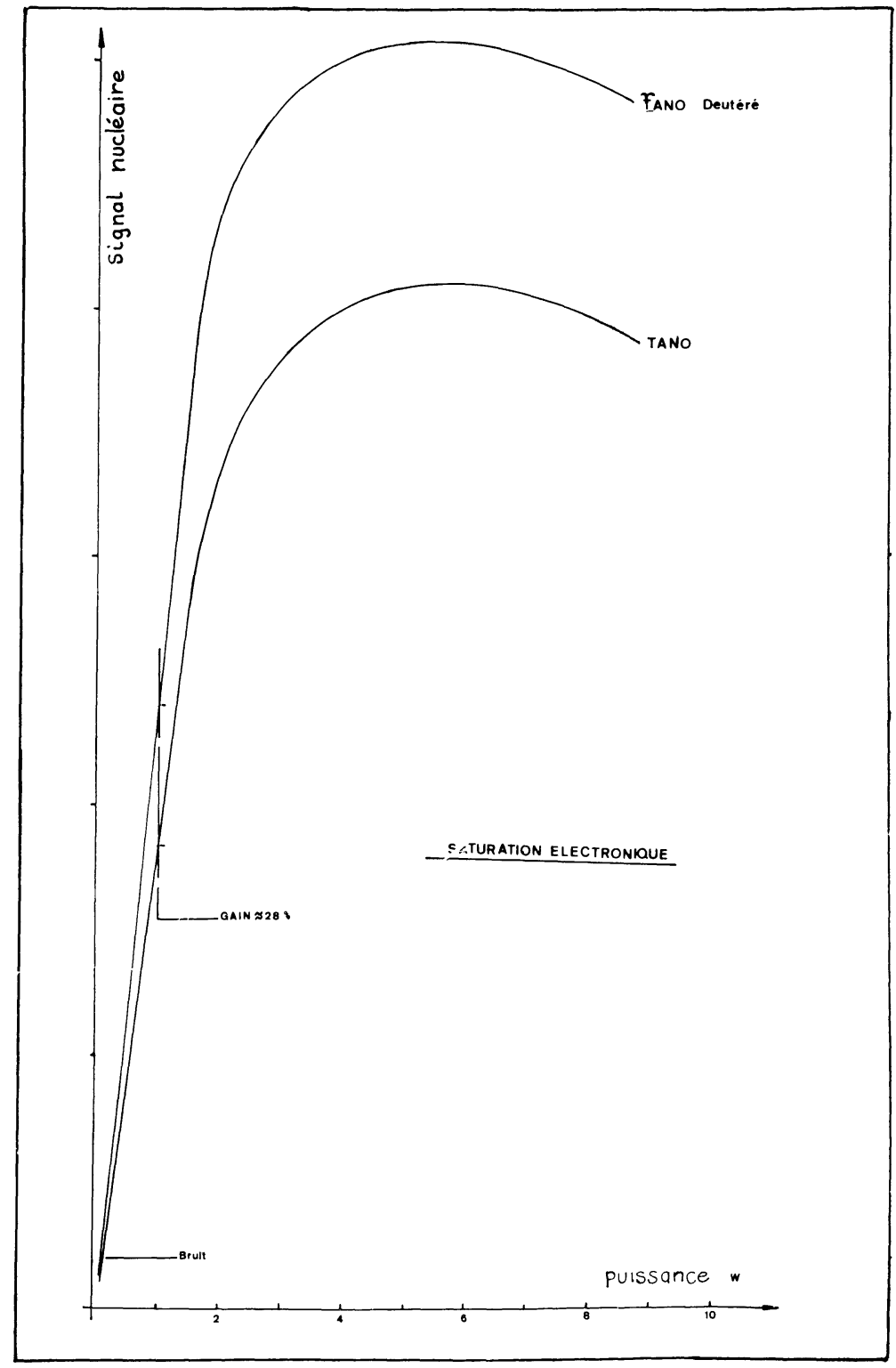

FIG. 7.

\section{BIBLIOGRAPHIE}

[1] Polarisation nucléaire par effet Overhauser dans les solutions d'ions paramagnétiques. Note de ABRAGAM (A.), Combrisson (J.) et Solomon (L.), présentée par PERRIN (F.).

[2] Étude de la relaxation d'un ion paramagnétique par observation des signaux de résonance nucléaire, par LANDESMan (A.), C.E.N., Saclay, J. Physique Rad., 1959, 20, 537.

[3] Effets des solvants sur les spectres U.V. et R.P.E. Mobilité conformationnelle des radicaux. BRIÈRE (R.), LEMAIRE (H.) et RASSAT (A.), Tetrahedon Letters, 1964, 27, 1775-1780. 